http://dx.doi.org/10.32929/2446-8355.2020v29n2p256-273

\title{
RESISTÊNCIA À PENETRAÇÃO EM SOLO SOB PASTAGEM DEGRADADA
}

Geovani José Silva ${ }^{1}$, João Carlos de Souza Maia ${ }^{2}$, Mariano Martinez Espinosa, Daniel Dias. Valadão Júnior $^{3 *}$, Aloísio Bianchini ${ }^{2}$, Franciele Caroline de Assis Valadão ${ }^{3}$

\footnotetext{
${ }^{1}$ Professor do Instituto Federal de Educação, Ciência e Tecnologia de Mato Grosso, Campus Cuiabá-MT.

${ }^{2}$ Professor da Universidade Federal de Mato Grosso, Campus Cuiabá-MT.

${ }^{3}$ Professor do Instituto Federal de Educação, Ciência e Tecnologia de Mato Grosso, Campus Campo Novo do Parecis-MT. *E-mail do autor correspondente: daniel.valadao@cnp.ifmt.edu.br
}

Recebido: 03/10/2019; Aceito: 02/06/2020

RESUMO: Este trabalho teve por objetivo, avaliar a estratificação do perfil da resistência à penetração (RP) em solo de textura média sob pastagem degradada, por meio da técnica de componentes principais. A penetrometria foi realizada em 120 pontos distantes $0,50 \mathrm{~m}$ do vértice de uma malha de $6 \times 10 \mathrm{~m}$, sendo dois pontos dispostos na linha de nível e dois acompanhando o desnível do terreno e, em outros 54 pontos radialmente distanciados em $0,125 \mathrm{~m}$. Os fatores foram extraídos por componente principais, rotacionados pelo método varimax e retidos conforme os critérios de Kaiser-Guttman e da análise paralela de Horn. As medidas de adequação de amostragem variaram de 0,733 a 0,852 . A maior variabilidade ocorreu nas camadas superficiais, com coeficientes de variação (CV) de até $58,20 \%$ contra CV de 13,38\% nas camadas intermediárias. Os maiores valores médios foram registrados entre 30 e $80 \mathrm{~mm}$ de profundidade, atingindo 5,55 MPa para ensaios realizados na linha de nível e 6,12 MPa quando acompanhado o desnível do terreno. A RP medida por um penetrógrafo se adequa ao tratamento estatístico da análise fatorial por componentes principais e o critério de Kaiser-Guttman possibilita modelos de AF melhores ajustados, para estratificar o perfil RP.

Palavras-chave: Compactação do solo. Componentes principais. Atributo físico. Penetrógrafo de solo.

\section{RESISTANCE TO PENETRATION IN SOIL UNDER DEGRADED PASTURE}

ABSTRACT: The objective of this work was to evaluate the stratification of the penetration resistance (PR) profile in medium texture soil under degraded pasture, using the main components technique. Penetrometry was performed at 120 points distant $0.50 \mathrm{~m}$ from the vertex of a $6 \times 10 \mathrm{~m}$ mesh, two points being arranged at the level line and two following the unevenness of the terrain and at another 54 points radially distanced at $0.125 \mathrm{~m}$. The factors were extracted by main components, rotated by the varimax method and retained according to Kaiser-Guttman criteria and Horn's parallel analysis. Sampling adequacy measures ranged from 0.733 to 0.852 . The greatest variability occurred in the superficial layers, with coefficients of variation (CV) of up to $58.20 \%$ versus $\mathrm{CV}$ of $13.38 \%$ in the intermediate layers. The highest mean values were recorded between 30 and $80 \mathrm{~mm}$ depth, reaching 5.55 $\mathrm{MPa}$ for tests performed at the level line and 6.12 MPa when accompanied by the unevenness 
of the terrain. The RP measured by a penetrograph is adequate for the statistical treatment of factorial analysis by main components, and the Kaiser-Guttman criterion allows better adjusted models of AF to stratify the RP profile.

Key words: Soil compaction. Principal components. Physical atributes. Soil penetrograph

\section{INTRODUÇÃO}

Dentre os diversos indicadores de qualidade física em solos manejados, direta ou indiretamente relacionados ao estado compactação do solo, a resistência à penetração (RP) medida por um penetrômetro tem sido a metodologia mais aplicada e tem se mostrado eficiente na localização e quantificação de camadas adensadas ou de restrição mecânica ao pleno crescimento radicular das plantas (TORMENA; ROLOFF, 1996; OLIVEIRA FILHO et al., 2016). Isso, em razão da rapidez, facilidade, baixo custo e carácter pouco invasivo na obtenção dessa variável (CAMARGO; ALLEONI, 1997; OLIVEIRA et al., 2007), além da considerável correlação entre a resistência do solo à penetração e o desenvolvimento das raízes encontrada por diversos pesquisadores de Taylor et al. (1966) a Costa et al. (2012). Valadão Junior et al. (2016) usando análise de componentes principais, elegeram a RP dentre um grupo de diversas variáveis, como a variável capaz de melhor explicar o efeito de diferentes implementos e do tempo na qualidade física do solo.

A RP depende da textura e condição estrutural do solo (TARDIEU, 1994), do tipo e teor de mineral de argila (CORRÊA et al., 2008), apresenta relação direta com a densidade e inversa com o conteúdo de água (TORMENA et al., 1998; CUNHA et al., 2002) e com carbono orgânico do solo (LUCIANO et al., 2010). Tudo isso, aliado ao caráter pontual e a elevada variabilidade vertical e horizontal da resistência à penetração, tem gerado polêmicas e controvérsias e dificultado a obtenção de valores críticos (WEIRICH NETO et al., 2006). O valor do nível crítico da resistência à penetração pode variar de 1,5 MPa (NESMITH, 1987) a 7,0 MPa como sugerem Sene et al. (1985) para solos arenosos. Todavia, tem se aceito o valor de $2 \mathrm{MPa}$ proposto por Taylor et al. (1966) como o limite crítico de resistência à penetração (TORMENA et al., 1998).

A definição dos limites das camadas do solo, em que a média possa ser um bom parâmetro para representação das resistências à penetração em profundidade, é difícil e carregada de subjetividade (WEIRICH NETO et al., 2006). Essa definição tem sido feita a priori, arbitrada no bom senso e sem estudos prévios na maioria dos trabalhos. Poucos estudos têm sido realizados, no sentido de reduzir essa subjetividade e estabelecer uma análise mais específica na definição dessas camadas. Excetuam-se os trabalhos de Weirich Neto et al. (2006) e Colet et al (2008) que utilizaram da técnica multivariada de análise fatorial (AF), na estratificação do perfil de resistência do solo à penetração em uma área sob plantio direto e sob pastagem respectivamente, baseando-se no que fizeram Stelluti et al. (1998) para diferentes sistemas de preparos do solo.

A análise fatorial (AF) é um conjunto de técnicas estatísticas multivariadas cujo objetivo é obter um reduzido número de fatores a partir da atribuição de escores, que 
ponderam as respostas de variáveis altamente correlacionadas no conjunto de variáveis originais, com a menor perda possível de informações (MARÔCO, 2010).

A verificação da fatorabilidade da matriz de dados deve ser o primeiro passo a ser realizado, verificando se a matriz de correlações populacionais estimada por $\mathrm{R}$ difere significativamente da matriz identidade (I). Aplica-se para isso, o teste de esfericidade de Bartlett e ou, se avalia a qualidade das correlações entre as variáveis, por meio da estatística de Kaiser-Meyer-Olkin (KMO) (MARÔCO, 2010).

A definição errônea do número de fatores a extrair na AF distorcerá os resultados da análise e conduzirá à reprodução de padrões e interpretações pobres. Dentre os vários critérios para definição do número de fatores a extrair, o critério da raiz latente maior que a unidade, ou critério de Kaiser-Guttman é o mais utilizado (HAIR JÚNIOR et al., 2009). Todavia, esse critério se mostrou adequado somente em $22 \%$ dos casos, enquanto que o critério baseado na análise paralela de Horn (1965) foi considerado adequado em 92\% dos casos, em estudo conduzido por Zwick e Velicer (1986).

Assim, apoiado na hipótese de que a resistência do solo à penetração em profundidade apresenta estrutura de interdependência, sendo a análise fatorial por componentes principais o tratamento estatístico necessário para estratificar seu perfil considerando tal estrutura, realizou-se este trabalho com os objetivos de: verificar a adequação dos dados do perfil de resistência à penetração em solo sob pastagem degradada, ao tratamento estatístico da análise fatorial por componentes principais; testar os critérios de Kaiser-Guttman e da análise paralela de Horn para retenção de fatores e, caracterizar o perfil de resistência de um Latossolo Vermelho sob pastagem degradada, definindo camadas de comportamento similar da resistência, aplicando a técnica multivariada da análise fatorial por componente principais, com rotação ortogonal.

\section{MATERIAL E MÉTODOS}

A pesquisa foi realizada no Instituto Federal de Educação Ciência e Tecnologia do Estado de Mato Grosso (IFMT) Campus São Vicente, cuja sede situa-se no quilômetro 329 da rodovia BR 364, Vila de São Vicente da Serra, município de Santo Antônio do Leverger MT. A área de pastagem degradada (PD) se encontra inserida na folha cartográfica sob índice de nomenclatura SD21ZDIV3NODIV1C, sob as coordenadas geográficas 1549'20.83" S e 55²4'06.49" W no sistema WGS 1984. O solo foi classificado como Latossolo Vermelho Distrófico típico (EMBRAPA, 2013), de textura média com 59,63\% de areia, 31,56\% de argila e $8,81 \%$ de silte.

Os ensaios de penetrometria foram realizados numa área sob pastagem de Urochloa brizantha explorada de forma extensiva, com mínimos tratos de manutenção e sem recuperação a mais de dez anos. Apresentava sintomas de degradação severa, como baixa produção de forragem, baixo índice de cobertura do solo, especialmente no período seco e presença de plantas invasoras.

A resistência do solo à penetração $(\mathrm{RP})$ foi medida utilizando um penetrógrafo robusto, desenvolvido por Bianchini et al. (2002), em conformidade com o padrão ASAE para 
penetrômetros de uso agrícola. O penetrógrafo foi acoplado a uma estrutura para deslocamento na área experimental, que permitia seu nivelamento, deslocamento horizontal em direções ortogonais, com fixação em pontos precisos (Figura 1).

O esquema amostral constituiu-se de uma malha de 30 vértices espaçados de $6 \times 10 \mathrm{~m}$. Em cada vértice foram realizados dois ensaios de penetrometria na linha de nível do terreno (EN) e dois acompanhando o desnível (ED). Os pontos ensaiados distanciavam 0,50 $\mathrm{m}$ do vértice da malha. Foram realizados, ainda, ensaios em 54 pontos, distribuídos em três sítios com 18 pontos espaçados radialmente em 0,125 m e dispostos em duplos hexágonos, referidos como ensaios adensados (EA). A finalidade desses foi avaliar a variabilidade horizontal da RSP em escala reduzida (Figura 1).
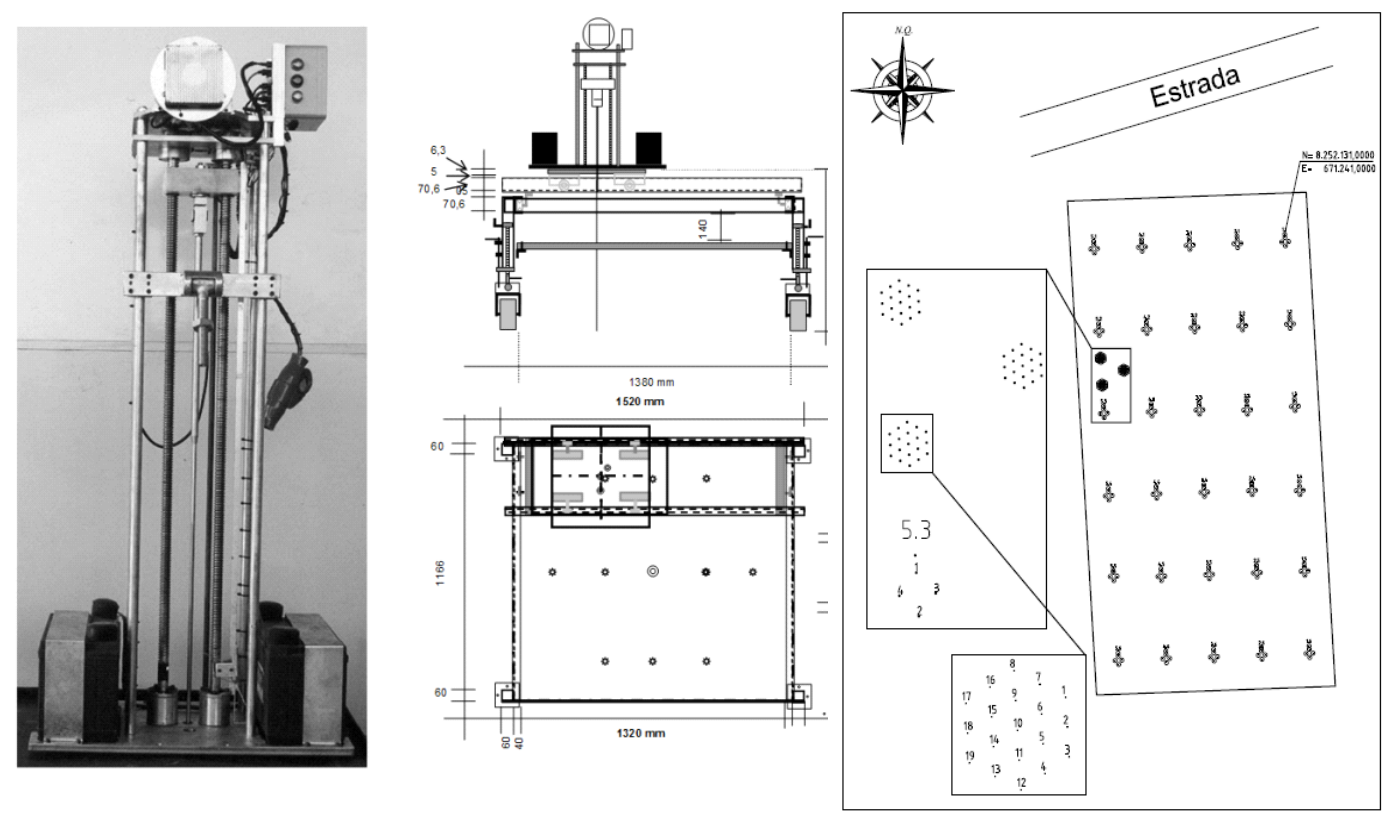

Figura 1. Penetrógrafo eletroeletrônico de Bianchini et al. (2002), estrutura para acoplamento e mobilidade e croquis da área experimental com a disposição dos pontos de ensaios da resistência do solo à penetração. Electro-electronic penetrograph by Bianchini et al. (2002), structure for coupling and mobility and sketches of the experimental area with the arrangement of the test points of soil resistance to penetration.

Fonte: Autoria própria. Own authorship

O penetrógrafo registrava valores de RP a cada 2,5 $\mathrm{mm}$ de aprofundamento do conjunto haste/cone até uma profundidade de $300 \mathrm{~mm}$. Os dados obtidos foram dispostos em planilhas com 30 colunas, correspondentes a intervalos de profundidades de $10 \mathrm{~mm}$ de espessura do perfil estudado (variáveis independentes). Cada linha correspondia a um ensaio (casos) e foi de 174 linhas na planilha geral (PD_G), 54 linhas para planilha de ensaios adensados (PD_EA) e 60 linhas para as planilhas de ensaios na linha de nível do terreno (PD_EN) e acompanhando o desnível (PD_ED). Cada célula dessas matrizes de dados originais, continha o valor médio dos quatro registros de resistência do solo à penetração em MPa.

Os conjuntos de dados foram submetidos a estatísticas descritivas. A aderência à distribuição normal foi avaliada pelo teste de Kolmogorov-Smirnov, em um nível de 
significância $\propto=0,05$ e pelo coeficiente de assimetria de Pearson $|\mathrm{SK}| \leq 1,0$ (LEECH et al., 2005).

As matrizes de dados originais foram submetidas a análise fatorial exploratória em modo R, para obtenção das matrizes simétricas das correlações. A significância das correlações populacionais $\Pi$ estimadas por $\mathrm{R}$ foi avaliada pelo teste de esfericidade de Bartlett, que testa as hipóteses $H 0: \Pi=I$ contra $H 1: \Pi \neq I$, sendo a estatística do teste $X^{2}=-\left(N-2-\frac{2 p+5}{6}\right) \log |R|$ em que, $\mathrm{N}$ : tamanho da amostra; $\mathrm{p}$ : número de variáveis; $|\mathrm{R}|$ é o determinante da matriz de correlação. Rejeita-se $H 0$ se $X^{2} \geq x_{1-\infty(p(p-1) / 2}^{2}$ graus de liberdade. A adequação dos dados de RSP para aplicação do modelo de análise fatorial, também foi avaliada mediante aplicação do teste de Kaiser-Meyer-Olkin (KMO). A medida de adequação da amostra é a razão entre o quadrado das correlações totais e quadrado das correlações parciais, das variáveis analisadas. Adotou-se como critério para intepretação do resultado deste teste e tomada de decisão, a classificação proposta por Sharma (1996).

Para extração de fatores foi adotado o método das componentes principais e na definição do número de fatores a ser retido, testou-se o critério de Kaiser-Guttman (autovalor > 1) e o critério baseado na análise paralela (AP) de Horn (1965). Nesse critério são comparados os autovalores empíricos com os resultantes de 1000 simulações Monte Carlo. São retidos os fatores cujos autovalores sejam maiores que os autovalores dos dados simulados (REISE et al., 2000).

Com o objetivo de melhorar a interpretação da solução fatorial, aplicou-se o método da rotação ortogonal varimax. Nesse método, a variância dos quadrados dos pesos fatoriais de cada variável é maximizada, sob a restrição de que permaneçam inalteradas as comunalidades. Assim, uma variável original fica fortemente associada a um único fator.

A avaliação empírica da qualidade do ajuste do modelo fatorial foi feita comparando a matriz de correlação observada (R) com a estimada pelo modelo ( $\Pi)$. Considerou-se como ajuste satisfatório, quando da ocorrência de mais de 50\% dos resíduos inferiores a 0,05. Foi usado também o índice RMSR* (Root Means Square Residual), o índice é baseado na média das correlações não redundantes obtidas pela padronização das covariâncias observadas e as geradas pelo modelo. Considerou-se como falta de ajuste, valores de RMSR* > 0,1; bom ajuste $0,05<\mathrm{RMSR}^{*}<0,1$ e muito bom quando os valores de RSMR* < 0,05 (SILVA, 2012).

A estratificação do perfil de RSP foi feita, em virtude das cargas fatoriais obtidas nas matrizes rotacionadas. Considerou-se cada fator retido como representando um estrato. A espessura ou os limites de cada camada foi definida então, pelos intervalos de profundidade com carregamentos significativos (significância empírica) no referido fator. Tomou como significativa carga fatorial igual ou maior que a obtida pelo modelo derivado das recomendações de Hair Júnior et al. (2009), $l_{L i}^{*}=4,9123 n^{-0,476}$, em que $l^{*}{ }_{L i}$ é a menor carga fatorial rotacionada, significativa a $\alpha=0,05$, um nível de poder de $80 \%$ e para uma amostra de tamanho $n$. O processamento das análises foi realizado utilizando o programa estatístico SPSS versão 17 para Windows. 


\section{RESULTADOS E DISCUSSÃO}

Os valores médios da RSP em profundidade variaram entre 2,236 MPa na P300 de PD_EA a 6,199 MPa observados na P50 em PD_EE (Tabela 1). Esses valores elevados de RSP, provavelmente, se devem à consolidação do solo em consequência das cargas impostas pelo pisoteio animal, somado à considerável parcela de contribuição do efeito da coesão (CUNHA et al., 2002), em consequência do estado de ressecamento do solo, dado que no momento dos ensaios o conteúdo de água era de 44,2 \pm 3,94\% da máxima capacidade de retenção do solo (Silva, 2012). Estes valores médios de RP, observados em todo o perfil avaliado, superam os 2,0 MPa amplamente aceito como nível crítico para a resistência de solo à penetração (TORMENA et al., 1998). Cabe ressaltar, que essa condição de ressecamento do solo ocorre em seis a oito meses do ano nessa região como demonstraram Fenner et al. (2014) em balaço hídrico para três biomas do estado. Isso, pode agravar as condições limitantes ao crescimento radicular e potencializar os processos de degradação das pastagens. Muito embora, Silva et al. (2006) tenham constatado que as gramíneas do gênero Urochloa sejam tolerantes e até mesmo beneficiadas por certa elevação na densidade dos solos e por consequência da resistência à penetração.

Verifica-se que os maiores valores de RP ocorreram nas camadas, em torno de P50, fato que se explica em razão da concentração nessa camada, dos efeitos das cargas de pisoteio animal, concordando com Trein et al. (1991) que relataram aumento de 3,8 vezes nos valores de RP na camada de 0 a 0,07 m e Ortigara et al. (2014) que observaram níveis de RP impeditivos nessa camada, devido ao pastejo intensivo. Observando ainda, os dados constantes no Tabela 1, nota-se que tanto o maior dentre os menores (2,502 $\mathrm{MPa})$, quanto o maior entre os maiores valores médios de RSP foram registrados em PD_ED. Isso, provavelmente retrate o efeito de fluxos preferenciais e armazenamento de água no solo em função do declive, visto que, conforme Souza et al. (2004), a variabilidade diferenciada nos atributos físicos dos solos é condicionada por pequenas variações nas formas do relevo.

A variabilidade da RP foi maior nas camadas mais superficiais independente do conjunto de dados avaliados e como se esperava, o aumento no número de ensaios por unidade de área em PD_EA promoveu redução considerável nos coeficientes de variação (CV) da RP em todo o perfil avaliado. O menor CV da RP $(13,38 \%)$ foi observado na P130 de PD_EA, enquanto o maior CV, da ordem de 58,20\%, foi verificado na P10 de PD_EN. Esses valores de coeficientes de variação são ligeiramente menores que os CVs encontrados por Souza et. al. (2004) para Latossolo Vermelho distrófico sob cultivo de cana. A variabilidade da RP em superfície neste trabalho é menor que a metade da variabilidade observada por Arzeno et al. (2014) para o mesmo tipo de solo sob semeadura direta e na camada de $50 \mathrm{~mm}$ de profundidade, os CVs obtidos na presente pesquisa não chegam a um terço do $\mathrm{CV}$ da resistência obtido por Blainski et al. (2008) em LVd sob pastagem. 
Tabela 1. Valores médios e coeficiente de variação (CV) da resistência à penetração de um Latossolo Vermelho sob pastagem degradada (PD), por intervalo de profundidade (P) e posição de ensaio ${ }^{1}$. Mean values and coefficient of variation $(C V)$ of penetration resistance of a Latossolo Vermelho under degraded pasture $(D P)$, by depth range $(P)$ and test position ${ }^{l}$.

\begin{tabular}{|c|c|c|c|c|c|c|c|c|}
\hline \multirow{3}{*}{$\begin{array}{c}\mathrm{P} \\
(\mathrm{mm})\end{array}$} & \multirow{2}{*}{\multicolumn{2}{|c|}{$\frac{\text { PD_G }}{\mathrm{N}=174}$}} & \multirow{2}{*}{\multicolumn{2}{|c|}{$\begin{array}{l}\text { PD_EA } \\
\mathrm{N}=54\end{array}$}} & \multirow{2}{*}{\multicolumn{2}{|c|}{$\begin{array}{l}\text { PD_EN } \\
\mathrm{N}=60\end{array}$}} & \multicolumn{2}{|c|}{ PD_ED } \\
\hline & & & & & & & & \\
\hline & $\begin{array}{l}\text { Média } \\
\text { (Mpa) }\end{array}$ & CV\% & $\begin{array}{l}\text { Média } \\
\text { (Mpa) }\end{array}$ & CV\% & $\begin{array}{l}\text { Média } \\
\text { (Mpa) }\end{array}$ & CV\% & $\begin{array}{l}\text { Média } \\
\text { (Mpa) }\end{array}$ & CV\% \\
\hline 10 & 2,788 & 52,12 & 3,280 & 38,28 & 2,440 & 58,20 & 2,693 & 57,67 \\
\hline 20 & 4,250 & 39,40 & 4,692 & 31,37 & 3,878 & 42,30 & 4,223 & 42,79 \\
\hline 30 & 5,392 & 28,87 & 5,553 & 22,81 & 5,064 & 32,18 & 5,573 & 30,22 \\
\hline 40 & 5,858 & 24,02 & 5,841 & 18,28 & 5,624 & 28,36 & 6,090 & 24,74 \\
\hline 50 & 5,942 & 21,11 & 5,824 & 15,39 & 5,771 & 25,14 & 6,199 & 21,90 \\
\hline 60 & 5,827 & 19,33 & 5,694 & 14,61 & 5,657 & 24,11 & 6,092 & 18,83 \\
\hline 70 & 5,626 & 17,46 & 5,484 & 13,53 & 5,440 & 24,13 & 5,885 & 15,53 \\
\hline 80 & 5,379 & 17,76 & 5,249 & 13,85 & 5,167 & 23,92 & 5,678 & 14,78 \\
\hline 90 & 5,123 & 18,38 & 5,013 & 14,47 & 4,884 & 24,79 & 5,426 & 15,55 \\
\hline 100 & 4,883 & 19,31 & 4,773 & 14,39 & 4,668 & 25,64 & 5,166 & 17,32 \\
\hline 110 & 4,619 & 20,09 & 4,510 & 14,37 & 4,426 & 26,51 & 4,878 & 18,71 \\
\hline 120 & 4,377 & 20,32 & 4,266 & 13,63 & 4,212 & 25,70 & 4,617 & 20,28 \\
\hline 130 & 4,172 & 20,85 & 4,017 & 13,38 & 4,041 & 24,38 & 4,444 & 21,04 \\
\hline 140 & 3,962 & 21,31 & 3,760 & 13,98 & 3,864 & 23,42 & 4,256 & 23,09 \\
\hline 150 & 3,765 & 22,18 & 3,534 & 14,42 & 3,706 & 23,35 & 4,054 & 25,22 \\
\hline 160 & 3,607 & 23,21 & 3,393 & 16,03 & 3,589 & 24,76 & 3,840 & 26,35 \\
\hline 170 & 3,483 & 24,17 & 3,288 & 17,90 & 3,488 & 25,57 & 3,670 & 27,16 \\
\hline 180 & 3,361 & 24,83 & 3,173 & 19,35 & 3,391 & 25,92 & 3,516 & 27,80 \\
\hline 190 & 3,240 & 24,56 & 3,059 & 19,60 & 3,287 & 25,43 & 3,369 & 27,35 \\
\hline 200 & 3,125 & 23,57 & 2,973 & 18,18 & 3,159 & 24,64 & 3,234 & 26,19 \\
\hline 210 & 3,003 & 22,08 & 2,890 & 16,95 & 3,032 & 22,90 & 3,083 & 25,25 \\
\hline 220 & 2,900 & 20,86 & 2,815 & 16,37 & 2,919 & 21,36 & 2,957 & 23,53 \\
\hline 230 & 2,826 & 20,52 & 2,748 & 16,86 & 2,852 & 21,66 & 2,870 & 22,26 \\
\hline 240 & 2,757 & 20,94 & 2,682 & 17,46 & 2,794 & 22,70 & 2,792 & 22,26 \\
\hline 250 & 2,681 & 21,71 & 2,613 & 17,75 & 2,711 & 23,59 & 2,717 & 23,30 \\
\hline 260 & 2,618 & 22,80 & 2,537 & 18,22 & 2,634 & 25,91 & 2,681 & 23,60 \\
\hline 270 & 2,544 & 23,03 & 2,456 & 18,85 & 2,556 & 26,20 & 2,618 & 23,66 \\
\hline 280 & 2,482 & 23,04 & 2,377 & 20,26 & 2,492 & 26,00 & 2,574 & 22,62 \\
\hline 290 & 2,430 & 23,19 & 2,299 & 21,44 & 2,433 & 25,64 & 2,550 & 21,90 \\
\hline 300 & 2,378 & 23,52 & 2,236 & 22,74 & 2,385 & 25,65 & 2,502 & 21,24 \\
\hline
\end{tabular}

Nota: ${ }^{1}$ PD_G: pastagem degradada geral: (PD_EA + PD_EN + PD_ED) respectivamente, ensaios adensados, na linha de nível e na linha de declive da área. Note: ${ }^{1} P D \_G$ : general degraded pasture: $\left(P D \_E A+P D \_E N+\right.$ $\left.P D \_E D\right)$ respectively, thickened trials at the level line and the slope line of the area.

Fonte: Autoria própria. Own authorship. 
Tabela 2. Medidas de adequação dos dados do perfil da resistência à penetração de um Latossolo Vermelho sob pastagem degradada à técnica multivariada de análise fatorial. Measurements of adequacy of the penetration resistance profile data of a Latossolo Vermelho under degraded pasture to multivariate factor analysis technique

\begin{tabular}{lccccc}
\hline \multirow{2}{*}{ Matriz $^{\mathrm{a}}$} & \multicolumn{2}{c}{ Teste de esfericidade Bartlett } & \multicolumn{2}{c}{ MSA $^{\mathrm{b}}$} \\
\cline { 2 - 6 } & Aprox, $\chi^{2}$ & Sig, & KMO $_{\mathrm{G}}$ & $\mathrm{KMO}_{(\mathrm{i}) \min ,}$ & KMO $_{(\mathrm{i}) \max ,}$ \\
\hline PD_G & 15674,05 & 0,000 & 0,852 & 0,656 & 0,902 \\
PD_EA & 4342,69 & 0,000 & 0,773 & 0,570 & 0,874 \\
PD_EN & 5265,46 & 0,000 & 0,788 & 0,518 & 0,872 \\
PD_ED & 5143,78 & 0,000 & 0,773 & 0,550 & 0,878 \\
\hline
\end{tabular}

Nota: ${ }^{a}$ G: matriz geral de dados [EA, EN, ED (N= 174)]; EA: ensaios adensados (N= 54); EN: ensaios na linha de nível do terreno e ED: ensaios na linha de declive com 60 casos cada, b Medida de adequação da amostragem de Kaiser-Meyer-Olkin global e, mínimo e máximo de i,..., p variáveis. Note: ${ }^{a}$ G: general data matrix [EA, EN, $E D(N=174)] ; E A$ : thickened assays $(N=54) ; E N$ : ground level line tests and ED: slope line tests with 60 cases each, $b$ Global Kaiser-Meyer-Olkin sampling adequacy measure and minimum and maximum $i, \ldots, p$ variables.

Fonte: Autoria própria. Own authorship.

As medidas de adequação da amostragem de Kaiser-Meyer-Olkin para cada matriz $\left(\mathrm{KMO}_{\mathrm{G}}\right)$ permite classificar a planilha geral de dados como de boa adequação e como média adequação à aplicação da análise fatorial, os demais conjuntos de dados, em conformidade com os critérios de Sharma (1996). A particularização do teste de KMO para cada variável, obtida da diagonal da matriz de correlações anti-imagem (que é a matriz das correlações parciais entre as variáveis) e designada por KMO (i) apresentou valores que variaram de 0,518 a 0,902. Isso, avalizou a inclusão de todos os intervalos de profundidades (P10 a P300) na análise fatorial, visto que somente valores de $\mathrm{KMO}_{\mathrm{i}}$ inferiores a 0,5 podem suscitar a retirada da variável do modelo de AF de acordo com Hair Júnior et al. (2009).

Constata-se ao observar a Figura 2, que o número de fatores retidos ao se adotar o critério de Kaiser-Guttman foi o mesmo para as quatro matrizes de dados analisadas e, por este critério reteve-se um ou dois fatores a mais, que quando se considera o critério da análise paralela de Horn (AP). Os três ou quatro fatores a reter definidos pelo critério da AP, se equivale ao mesmo número de fatores que segundo Weirich Neto et al. (2006) explicaram $85,8 \%, 87,3 \%$ e $94,4 \%$ da variância da RP em profundidade, em um Latossolo sob semeadura direta. Esses valores de variância acumulada explicada são similares aos aqui encontrados, embora, só se tenha superado $90 \%$ de variância explicada, nos modelos com cinco fatores retidos (critério de Kaiser-Guttman). Cinco fatores foi também foi o número encontrado por Colet et al (2008) para explicar 93,6\% da variância da resistência à penetração de um Latossolo Vermelho Amarelo sob pastagem. 

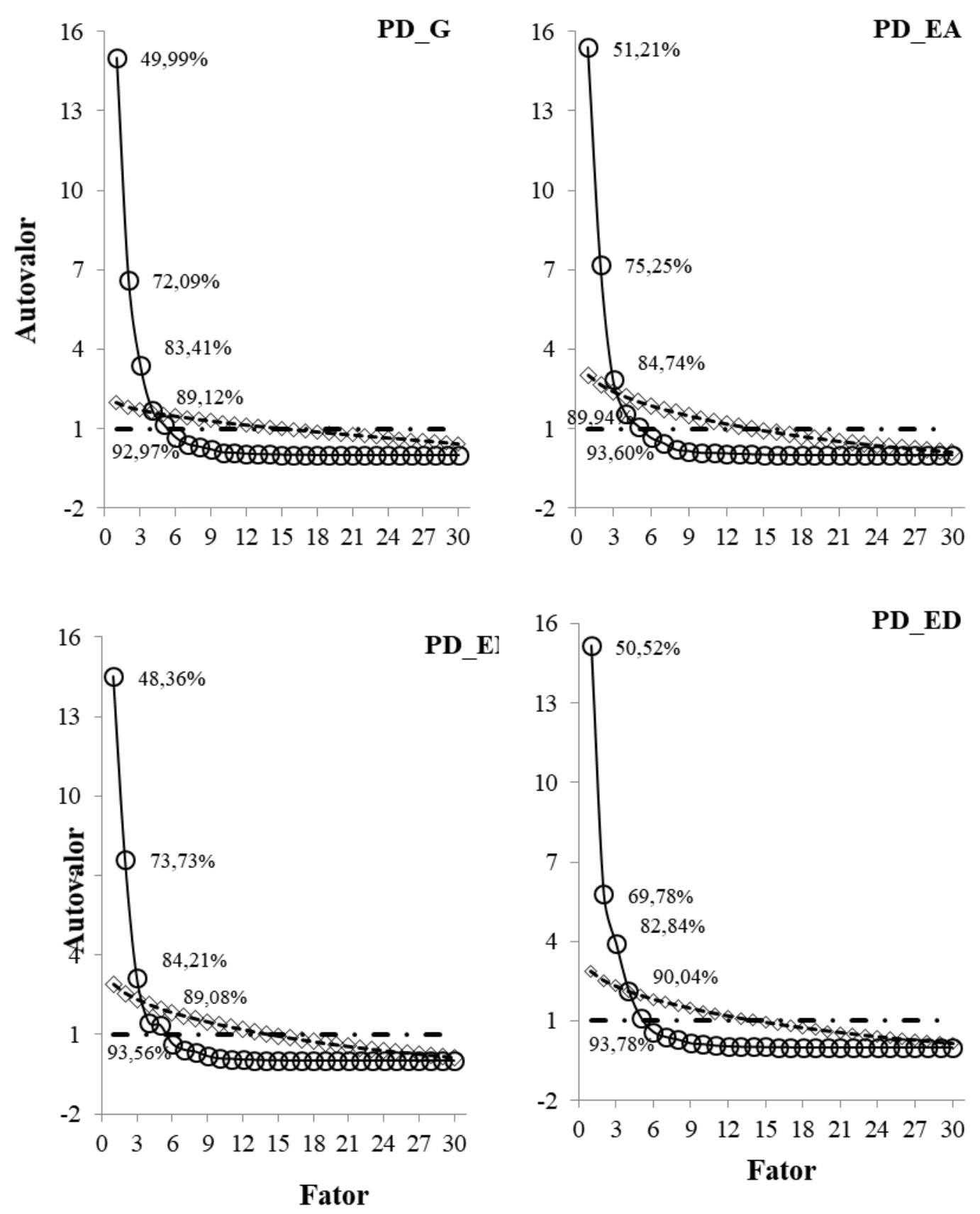

Figura 2. Autovalores empíricos (०), obtidos por simulação Monte Carlo $(\diamond)$ e variância acumulada (\%) explicada por fatores retidos pelos critérios de Kaiser-Guttman (・ - •) e análise paralela de Horn, da resistência à penetração em Latossolo sob pastagem degrada (PD), submetida à análise fatorial exploratória, conjuntamente (PD_G), em ensaios adensados (PD_EA), ensaios na linha de nível terreno (PD_EN) e acompanhando o declive (PD_ED). Empirical eigenvalues ( $(0)$ obtained by Monte Carlo simulation $(\diamond)$ and cumulative variance (\%) explained by factors retained by the Kaiser-Guttman criteria (- - -) and Horn's parallel analysis of the Latossolo penetration resistance under degraded pasture (PD), submitted to exploratory factor analysis, jointly (PD_G), in densified tests (PD_EA), ground level line (PD_EN) and following the slope (PD_ED).

Fonte: Autoria própria. Own authorship 
Os oito modelos fatoriais obtidos adotando os dois critérios de retenção de fatores, apresentam "bom" ou "muito bom" ajuste, conforme Marôco (2010). Os melhores ajustes foram obtidos pelos modelos com 5 fatores que apresentaram de 4 a $8 \%$ de resíduos superiores a 0,05 e raízes do erro quadrático médio (RMSR*) de 0,026 a 0,029 (Tabela 3). Enquanto os modelos com 4 e 3 fatores apresentaram qualidade de ajuste um pouco menor, com 17 a 44\% de resíduos, em módulo, maiores que 0,05 e RMSR* variando de 0,044 a 0,072 .

Tabela 3. Qualidade e ajuste dos modelos fatoriais encontrados para o perfil da resistência à penetração em Latossolo sob pastagem degradada, em função de critério para retenção de fatores e matriz de dados de entrada. Quality and fit of the factor models found for the Latossolo penetration resistance profile under degraded pasture, according to criteria for factor retention and input data matrix.

\begin{tabular}{|c|c|c|c|c|c|c|}
\hline Critério & Matriz $^{1}$ & $\begin{array}{c}\mathbf{N}^{\mathbf{o}} \\
\text { Fatores }\end{array}$ & $\begin{array}{c}\text { Autovalor } \\
\text { (mínimo) }\end{array}$ & $\begin{array}{c}\text { Variância } \\
\text { Explicada } \\
(\%)\end{array}$ & $\begin{array}{c}(\%)^{2} \\
|e|^{>0,05}\end{array}$ & $\mathbf{R M S R}^{* 3}$ \\
\hline \multirow{4}{*}{$\begin{array}{c}\text { Kaiser } \\
\text { Autovalor }>1\end{array}$} & G & 05 & 1,15 & 93,0 & 8,00 & 0,0291 \\
\hline & EA & 05 & 1,10 & 93,6 & 6,66 & 0,0282 \\
\hline & $\mathrm{EN}$ & 05 & 1,34 & 93,6 & 7,00 & 0,0288 \\
\hline & ED & 05 & 1,12 & 93,8 & 4,00 & 0,0259 \\
\hline \multirow{4}{*}{ Análise paralela de Horn } & $\mathrm{G}$ & 04 & 1,71 & 89,1 & 19,00 & 0,0477 \\
\hline & EA & 03 & 2,85 & 84,7 & 37,70 & 0,0677 \\
\hline & EN & 03 & 3,14 & 84,2 & 44,00 & 0,0718 \\
\hline & ED & 04 & 2,16 & 90,0 & 17,00 & 0,0444 \\
\hline
\end{tabular}

Nota: ${ }^{1}$ G: matriz geral de dados [EA+EN, ED $(\mathrm{N}=174)$ ]; EA: ensaios adensados $(3 \times 18$ ensaios dispostos radialmente a $0,125 \mathrm{~m} \mathrm{~N}=54$ ); EN: ensaios linha de nível ao declive ou de semeadura e EE: ensaios na linha de declive ou entre linha de semeadura com 60 casos; ${ }^{2}$ |e| Resíduo em módulo; ${ }^{3}$ Raiz do erro quadrático médio. Note; ${ }^{l}$ G: general data matrix [EA $\left.+E N, E D(N=174)\right]$; EA: densified assays ( $3 x 18$ assays radially arranged at $0.125 \mathrm{~m} \mathrm{~N}=54$ ); EN: slope level or seeding line tests and EE: slope line or seeding line trials with 60 cases; ${ }^{2}$ $\mid$ and $\mid$ Module waste; ${ }^{3}$ Root of the mean square error.

Fonte: Autoria própria. Own authorship.

As cargas fatoriais e as comunalidades das 30 variáveis (profundidades P10 a P300), obtidas por AF/ACP e rotacionadas pelo método varimax, com fatores retidos conforme determinado pelos critérios de Kaiser-Guttman (K) e da análise paralela de Horn (AP) se encontram resumidas na Tabela 4. Importante salientar que as cores das fontes relativas aos valores numéricos de cada carga fatorial, têm função de facilitar a identificação visual das diferentes camadas da RP, bem como, permitir avaliar suas espessuras e posições no perfil do solo, em função das diferentes matrizes de dados de entrada e critérios de retenção dos fatores São fortes as correlações entre as profundidades que formam uma camada e o respectivo fator, como demonstram as cargas de valores que se aproximam da unidade. O valor médio dos carregamentos fatoriais em todos os conjuntos de dados e nas diferentes soluções foi de 0,840 . Esse carregamento fatorial se equivale ao verificado por Arcoverde et al. (2015) para atributos físicos utilizados na definição de índice de qualidade física de solo agrícola. Cargas fatoriais, cerca de $2 \%$ menores em média, foram observadas para os modelos em ensaios adensados, onde também se verificam o maior número de cargas desprovidas de significância empírica. No entanto, a única variável com carga fatorial não significativa $(0,63)$ e 
comunalidade $(0,457)$ inferior a 0,5 foi P10 na solução com 3 fatores em PD_EA. Isso, motivaria excluir essa variável do modelo (MENDONÇA et al. 2007; HAIR JÚNIOR et al., 2009) e apesar de Murakami e Cruz (2004) não verem assegurada a associação de variável a um constructo quando esta possua carga entre 0,5 e 0,7 , no entanto, o valor da carga se aproxima dos 0,65 adotados como relevante por Arcoverde et al. (2015). Ainda, a baixa comunalidade expressa pela profundidade P10, parece resultar da retenção de número insuficiente de fatores no caso, visto que, na solução mais parcimoniosa com 5 fatores retidos, a variável passa carregar no Fator 4 com carga de 0,895 e tem sua comunalidade elevada para 0,839 (Tabela 4).

O maior número de cargas não significativas observadas em PD_EA se deve ao número de casos (N) muito próximo do mínimo sugerido por Hair Júnior et al. (2009) que seria 50 casos, pois os mesmos autores sugerem 100 casos para obtenção de resultados robustos em AF. Entretanto, tais carregamentos fatoriais são todos muito próximos e até mesmo superam alguns critérios de relevância de cargas fatoriais adotados, como os 0,7 de Mendonça et al. (2007) e Lorentz e Nunes (2013) e o critério de Arcoverde et al. (2015) já citado. Além disso, tais variáveis (profundidades) apresentam comunalidades suficientemente elevadas para assegurarem sua associação ao fator conforme Johnson e Wichern (2005).

A observação da Tabela 4, permite verificar ainda, que os intervalos de profundidade com os maiores carregamentos em um fator, correspondem às profundidades do terço médio dessa camada do solo definida por esse fator. Decréscimo nos valores dos carregamentos fatoriais ocorrem ao se afastar do terço médio de cada camada, retratando a característica transicional das subcamadas na estratificação do perfil da RP. Os limites das camadas foram então determinados pelo carregamento fatorial de maior valor nominal, desses intervalos de profundidade de transição, visto que os mesmos geralmente carregaram em mais de um fator. Embora Marôco (2010) afirme que esse fato deponha contra a ortogonalidade dos fatores, optou-se como Magalhães (2008) por manter tais variáveis na análise. Ponderou-se a significância empírica das cargas fatoriais desses intervalos de profundidades, dado a fragilidade de fundamentação estatística para esse critério, por se tratarem de variáveis concretas e que, devido a característica de transição, essa subcamada pode apresentar diferenças e similitudes tão tênues que a poderiam associar tanto à camada superior quanto ao fator representativo da camada imediatamente inferior. Ainda é possível constatar a formação de uma camada composta apenas pelas profundidades P200 e P210 na solução fatorial pelo critério de Kaiser-Guttman em PD_EA. As cargas fatoriais nessa camada apesar de elevadas não são significativas, verificando ainda que o autovalor associado ao quinto fator e muito próximo de 1,00 levanta-se a possibilidade de avaliar uma solução com a retenção de 4 fatores. Assim, muito provavelmente P200 e P210 se associariam as camadas 3 e 4 respectivamente. 
Tabela 41. Carregamento fatorial (CF) e Comunalidade (Com) após rotação varimax, da resistência do solo à penetração por intervalo de profundidade $(\mathrm{P})$, em função da matriz de dados de entrada e critério de retenção de fatores de Kaiser-Guttman (K) e análise paralela (AP) em solo sob pastagem degradada. Factorial loading (CF) and Commonality (Com) after varimax rotation of soil resistance to depth interval penetration $(P)$, as a function of input data matrix and Kaiser-Guttman factor retention criteria $(K)$ and parallel analysis $(A P)$ in soil under degraded pasture

\begin{tabular}{|c|c|c|c|c|c|c|c|c|c|c|c|c|c|c|c|c|}
\hline \multirow{3}{*}{$\begin{array}{c}\mathrm{P}^{1} \\
(\mathrm{~mm})\end{array}$} & \multicolumn{4}{|c|}{ Geral } & \multicolumn{4}{|c|}{ Ensaios adensados } & \multicolumn{4}{|c|}{ Ensaios linha de Nível } & \multicolumn{4}{|c|}{ Ensaios linha de Declive } \\
\hline & \multicolumn{2}{|c|}{$\mathrm{K}$} & \multicolumn{2}{|c|}{$\mathrm{AP}$} & \multicolumn{2}{|c|}{$\mathrm{K}$} & \multicolumn{2}{|c|}{$\mathrm{AP}$} & \multicolumn{2}{|c|}{$\mathrm{K}$} & \multicolumn{2}{|c|}{$\mathrm{AP}$} & \multicolumn{2}{|c|}{$\mathrm{K}$} & \multicolumn{2}{|c|}{40} \\
\hline & 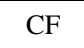 & , & & & $F_{2}$ & & $\mathrm{CF}$ & & $\mathrm{SF}$ & $C o m$ & SE & 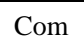 & $\mathrm{CF}_{\mathrm{S}}$ & Com & $\mathrm{CF}$ & Com \\
\hline 10 & & & & & & & & & 25 & & & & 923 & & 780 & 629 \\
\hline 20 & & 081 & 0002 & 0824 & 34 & 0,796 & 0,802 & 3 & 935 & 062 & 0 & 76 & ,931 & (20 &, 884 & ,79 \\
\hline 30 & & & & & & & & & 93 & & & & 88 & & & 905 \\
\hline 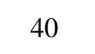 &, 773 & 0021 & 0,926 & 0012 & 745 & 0011 & 6 & 1 & 746 & 0015 & 0,918 & 04 &, 753 & 0,918 &, 946 & 01 \\
\hline 50 & & 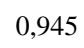 & 857 & & 759 & & & & 371 & & & & . & & & 88 \\
\hline 60 & & 070 & 0,760 & 0 ० & 0,860 & & 0,914 & & 4 & 0,915 & 0,140 & & 0,922 & 0,956 & ) & 79 \\
\hline 70 & 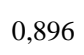 & 947 & 0,668 & 0,853 & 923 & 0,863 & 0,87 & 864 & 907 & 61 & $0,65^{\mathrm{NS}}$ & 703 &, 892 & 0,934 & .714 & 863 \\
\hline 80 & & קחק & & & 08 & & 0,1 & & ,797 & - & & 23 & 0,791 & 0 & 4 &, 870 \\
\hline 9 & ,752 & 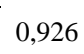 & 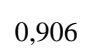 & 0,926 & ( & 0,924 & $0,66^{\mathrm{NS}}$ & 0 & , & s. & 0,000 & , & $x_{2}$ & 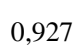 & , & 0,924 \\
\hline 100 & 870 & 941 & 014 & 918 & $0,73^{\mathrm{NS}}$ & 34 & & 6 & 0,918 & 0.05 & 0,905 & 57 & 0,869 & 0,947 &, 909 &, 93 \\
\hline 1 & & $0=0$ & 0,849 & & 7 & & & & 0,951 & & & & 0,910 & & 0,850 & 0,913 \\
\hline 120 & 06 & 050 & 0763 & os & 0,814 & 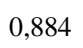 & 0,856 & 0,901 & 0,920 & 0,972 & 0,863 & 25 & 0,865 & 0,943 &, 741 & 0884 \\
\hline 130 & & م & 0,688 & & 0,895 & & & & 840 & & & & 0,779 & 0,944 & & 0,897 \\
\hline 140 & 597 & 005 & 0,719 & 0,904 & 0 & 212 & 0 & 0 & $0,69^{\mathrm{NS}}$ & 0,913 & 0,80 & 0,899 & $\overline{0.68}$ & 0,94 & 3 & 0,91 \\
\hline 15 & & & & & & & & & $0,68^{\mathrm{NS}}$ & 0,891 & 0,710 & 0,877 & 0,790 & 0,9 & 856 & 0,93 \\
\hline 10 & & & & & & & & & 3 & & $0,69^{\mathrm{NS}}$ & & 0,864 & & 0,895 & 0,953 \\
\hline 170 & & 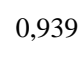 & 056 & 924 & 866 & & 0 & & 835 & & & & 0,893 & & 7 & 95 \\
\hline 180 & & 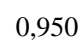 & 0,865 & & 0,766 & & 0,7 & & . & , & & & 7 & 0,966 & 0,912 & , \\
\hline 190 & & & & & $0,66^{\mathrm{NS}}$ & & $0,73^{\mathrm{NS}}$ & & 854 & & & & 0,908 & 0,9 & 900 & ,957 \\
\hline 200 & & & & & 0,66 & & $0,64^{\mathrm{N}}$ & & 0,861 & & & & 0,877 & & & 0,93 \\
\hline 210 & & & 0,789 & 0,898 & $0,64^{\mathrm{NS}}$ & & $0,64^{\mathrm{NS}}$ & & 0,869 & & & & 818 & &, 786 & 0.91 \\
\hline 220 & & & , & & $0,60^{\mathrm{NS}}$ & & 0,71 & & 0,826 & - & 0,859 & & 0,736 & 0,945 & 0,701 & 0,925 \\
\hline 230 & & & 0,700 & & $0,70^{\mathrm{NS}}$ & & 070 & &, 723 & 0,072 & & & 5 & & & 0,93 \\
\hline 240 & & & 0,8 & & & & & & & & 2 & & 0,847 & 0,941 &, 860 & 0,941 \\
\hline & & & & & & & & & & & & & & & & 0,929 \\
\hline 260 & & & & & & & & & & & & & 0,942 & & 933 & 0,91 \\
\hline 270 & or & > & תרס & م & 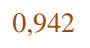 & ברב & (2000 & 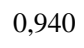 & 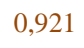 & Or & م & ( & 3 & 0,960 & 3 & 0,930 \\
\hline 28 & & & & & & & & & 4 & & & & 36 & 1 & 0,934 & 0,9 \\
\hline 290 & & & & & & & & & & & & & 6 & & 4 & 0,879 \\
\hline 300 & 860 & ,834 & 865 & 833 & 901 & 0,796 & 0,871 & 0,837 &, 894 & 0,907 & 0,894 & 0,803 & 0,797 & 0,798 & 0,814 & 0,796 \\
\hline
\end{tabular}

Nota: ${ }^{N S}$ Carga fatorial sem significância empírica (HAIR et al., 2009), ${ }^{1}$ P: intervalo de profundidade. Note:

${ }^{N S}$ Factorial load without empirical significance (HAIR et al., 2009), ${ }^{1} \mathrm{P}$ : depth range

Fonte: Autoria própria. Own authorship.

$\mathrm{Na}$ Figura 3, são retratadas as curvas da resistência do solo à penetração, as espessuras e localização das camadas determinadas pela análise fatorial, bem como seus respectivos 
valores médios de RP. Essas curvas são de formato muito semelhantes para todos os conjuntos de dados (PD_G; PD_EA; PD_EN e PD_ED), apesar da aleatoriedade que caracteriza a distribuição horizontal das cargas resultantes do pisoteio animal em área de pastagem.
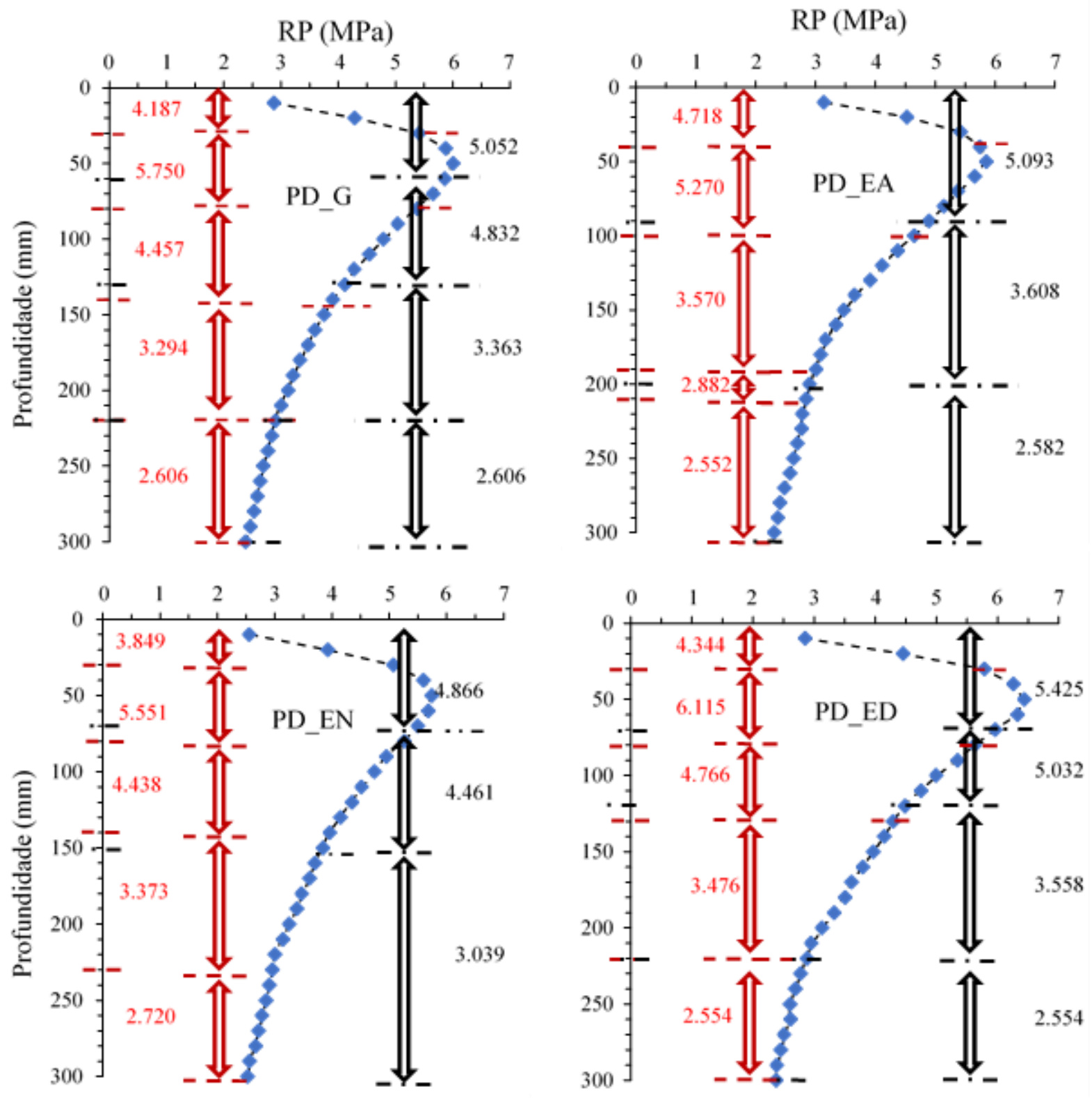

PD: Pastagem Degradada; G: matriz Geral de dados (EA + EN + ED); Ensaios de penetrometria adensados (radialmente distantes $12,5 \mathrm{~cm}$ ); ensaios na linha de nivel e, acompanhando a linha de maior declividade respectivamente; - - _ Limite definido / critério de Kaiser; _ - - Limite definido / Análise paralela de Horn:

Figura 3. Curvas da resistência do solo à penetração de um Latossolo Vermelho sob pastagem degradada, estratificada pela técnica multivariada da análise fatorial por componentes principais. Curves of soil resistance to penetration of a Latossolo Vermelho under degraded pasture, stratified by the multivariate technique of factor analysis by principal components.

Fonte: Autoria própria. Own authorship. 
Algumas posições no perfil do solo como as profundidades de 80, 140 e $220 \mathrm{~mm}$ se repetem como limite de camadas, independente do conjunto de dados e do critério que definiu o número de fatores, podendo sofrer variações de mais ou menos $10 \mathrm{~mm}$, semelhante ao erro de definição observado por Weirich Neto et al. (2006).

A condição local de PD_EA é refletida em alterações na espessura e posições das camadas formadas especialmente no terço médio do perfil. A estratificação do perfil de resistência obtida ao adotar critério de retenção de fatores menos restritivo parece mais adequada. Assim, aspectos dinâmicos da RP seriam melhor considerados. Pelo critério da análise paralela de Horn, os intervalos de profundidade da superfície até aproximadamente 80 mm são reunidos em uma única camada pela a AF. No entanto, ao se usar o critério de KaiserGuttman as profundidades P10, P20 e P30 em PD_G, PD_EN e PD_ED e mais P40 em PD_EA são reunidas em uma camada superficial. O desmembramento de profundidades que apresentam forte incremento na RP, de outras onde ocorre estabilização seguida de leve declínio nos valores dessa variável parece mais coerente. Colet et al. (2008) também identificaram uma estreita camada superficial de $20 \mathrm{~mm}$ em solo sob pastagem.

Os valores da RP na camada de 30 - 80 mm são em média de 11,7\% em PD_EA a $44,2 \%$ em PD_EN maiores que os registrados para a camada mais superficial $(0,0-30 \mathrm{~mm})$ justificando separação dessas camadas.

Analisando a Figura 3, verifica-se ainda, que as camadas com as maiores médias de RP e, portanto, mais limitadoras do crescimento de raízes são as camadas de $30-80 \mathrm{~mm}$. Nessas camadas os valores de RP são cerca de $12 \%$ maiores que as resistências médias nas camadas de maior impedimento, identificadas pelo critério da análise paralela (0-60, 0-90 e 0-70).

No terço médio e no terço mais inferior do perfil do solo não se observam maiores divergências entre as estratificações definidas pelos dois critérios. Em PD_EA a camada formada por P200 e P210 é provavelmente desnecessária e, a não divisão da camada de P150 a P300 em PD_EN na estratificação pelo critério da AP são as diferenças entre os 2 critérios, anotadas para o terço inferior do perfil. Diferenças nos valores médios da RP por camadas entre PD_EN e PD_ED da ordem de 3 a 13\% possibilitam pressupor o efeito do relevo na resistência do solo à penetração, corroborando Souza et al. (2004). De modo geral, a estratificação aqui obtida por AF com o critério de Kaiser-Guttman se assemelha aos resultados obtidos por Colet et al. (2008), para solo não escarificado sob pastagem.

\section{CONCLUSÃO}

Os dados da resistência do solo à penetração obtidos por um penetrógrafo foram adequados ao tratamento estatístico da análise fatorial por componentes principais.

O critério de Kaiser-Guttman é menos restritivo que o critério da análise paralela de Hon e possibilita modelos de análise fatorial melhores ajustados para estratificar o perfil da resistência do solo à penetração em pastagem de braquiária degradada. 
Os valores de RP sofrem forte incremento nos três ou quatro centímetros superficiais do solo sob pastagem e devem constituir camada própria não devendo ser computados no cálculo da RP média de camada mais espessa do perfil.

A camada de maior RP apresenta cerca de $50 \mathrm{~mm}$ e se localiza, aproximadamente, entre as profundidades de 30 e $80 \mathrm{~mm}$ no perfil de um solo sob pastagem degradada.

\section{REFERÊNCIAS BIBLIOGRÁFICAS}

ARCOVERDE, S. N. S.; SALVIANO, A. M.; OLSZEVSKI, N.; BEZERRA, S. M.; CUNHA, T.J.F.; GIONGO, V.; PEREIRA J. DE S. Qualidade física de solos em uso agrícola na região semiárida do estado da Bahia. Revista Brasileira de Ciência do Solo, Viçosa, v. 39, n. 5, p.1473-1482, 2015.

ARZENO, J. L.; VIEIRA, S. R.; SIQUEIRA, G. M.; GUEDES FILHO, O. Variabilidade espacial da resistência à penetração na superfície do solo em sistemas de manejo. Revista Brasileira de Ciências Agrárias, Jaboticabal, v. 9, n. 3, p.343-352, 2014.

BIANCHINI, A.; MAIA, J. C. M.; MAGALHÃES, P. S. G.; CAPPELLI, N.; UMEZU, C. K. Penetrógrafo eletrônico automático. Revista Brasileira de Engenharia Agrícola e Ambiental, Viçosa, v. 6, n. 2, p.332-336, 2002.

BLAINSKI, E.; TORMENA, C. A; FIDALSKI, J.; GUIMARÃES, R. M. L. Quantificação da degradação física do solo por meio da curva de resistência do solo à penetração. Revista Brasileira de Ciência do Solo, Viçosa, v. 32, n. 3, p.975-983, 2008.

CAMARGO, A. O.; ALLEONI, L. R. F. Compactação do solo e o desenvolvimento das plantas. Piracicaba: ESALQ, 1997. 132 p.

COLET, M.J.; SVERZUT, C.B.; WEIRICH NETO, P.H. Efeito da escarificação na resistência à penetração de um solo sob pastagem. Revista Brasileira de Engenharia de Biossistemas, Tupã, v. 2, n. 2, p.119-128, 2008.

CORREAA, M. M.; ANDRADE, F. V.; MENDONÇA, E. S.; SCHAEFER, C. E. G. R.; COSTA, T. T.; ALMEIDA, C. C. Ácidos orgânicos de baixo peso molecular e ácidos húmicos e alterações em algumas propriedades físicas e químicas de Latossolos, Plintossolo e Neossolo Quartzarênico. Revista Brasileira de Ciência do Solo, Viçosa, v. 32, n. 1, p.121$131,2008$.

COSTA, M. A. T.; TORMENA, C. A.; LUGÃO, S. M. B; FIDALSKI, J.; NASCIMENTO, W. G.; MEDEIROS, F.M. Resistência do solo à penetração e produção de raízes e de forragem em diferentes níveis de intensificação do pastejo. Revista Brasileira de Ciência do Solo, Viçosa, v. 36, n. 3, p.993-1004 2012.

CUNHA, J. P. A. R.; VIEIRA, L. B.; MAGALHÃES, A. C. Resistência mecânica do solo à penetração sob diferentes densidades e teores de água. Engenharia Agrícola, Jaboticabal, v. 10, n. 4, p.1-7, 2002.

EMPRESA BRASILEIRA DE PESQUISA AGROPECUÁRIA - EMBRAPA. Sistema brasileiro de classificação de solos. 3. ed. Rio de Janeiro: Embrapa, 2013. 376 p. 
FENNER, W.; MOREIRA, P. S. P.; FERREIRA, F. S.; DALLACORT, R.; QUEIROZ, T. M. D.; BENTO, T. S. Análise do balanço hídrico mensal para regiões de transição de CerradoFloresta e Pantanal, Estado de Mato Grosso. Acta Iguazu, Cascavel, v. 3, n. 1, p.72-85, 2014. HAIR JÚNIOR, J. F.; ANDERSON, R. E.; TATHAM, R. L.; BLACK, W. C. Análise multivariada de dados. 6. ed. Porto Alegre: Bookman; 2009. 688 p.

HORN, J. L. A rationale and test for the number of factors in factor analysis. Psychometrika, New York, v. 30, p.179-185, 1965.

JOHNSON, R. A.; WICHERN, D. W. Applied multivariate statistical analysis. Madison: Prentice Hall International, 2005. 593 p.

LEECH, N. L.; BARRETT, K. C.; MORGAN, G. A. SPSS for Intermediate Statistics: use and Interpretation. 2. ed. New Jersey: Lawrence Erlbaum Associate Publishers Mahwah. 2005. 240 p.

LORENTZ, L. H.; NUNES, U. R, Relações entre medidas de qualidade de lotes de sementes de arroz. Revista Ciência Agronômica, Jaboticabal, v. 44, n. 4, p.798-804, 2013.

LUCIANO, R. V.; BERTOL, I.; BARBOSA, F. T.; KURTZ, C.; FAYAD, A. J. Propriedades físicas e carbono orgânico do solo sob plantio direto comparados à mata natural, num Cambissolo Háplico. Revista Ciência Agroveterinária, Lages, v. 9, n. 1, p.9-19, 2010.

MARÔCO, J. Análise Estatística: com utilização do SPSS. 3 ed. Lisboa: Sílabo, 2010. 822 p.

MENDONÇA, O.; PÍPOLO, V.C.; GARBUGLIO, D. D.; FONSECA JUNIOR, N. D. S. Análise de fatores e estratificação ambiental na avaliação da adaptabilidade e estabilidade em soja. Pesquisa Agropecuária Brasileira, Brasília, v. 42, n. 11, p.1567-1575, 2007.

MURAKAMI, D. M.; CRUZ, C. D. Proposal of methodologies for environment stratification and analysis of genotype adaptability. Crop Breeding Applied Biotechnology, Viçosa, v. 4, p.7-11, 2004.

NESMITH, D. S. Soil compaction in double cropped wheat and soybean on Ultissol. Soil Science Society of America Journal, Madison, v. 51, n. 1, p.183-186, 1987.

OLIVEIRA FILHO, F. X.; MIRANDA, N. O.; MEDEIROS, J. F.; SILVA, P. C. M.; MESQUITA, F.O.; COSTA, T. K. G. Compactação de solo cultivado com cana-de-açúcar em Baía Formosa, Rio Grande do Norte. Revista Ceres, Viçosa, v. 63, n. 5, p.715-723, 2016.

OLIVEIRA, G. C.; SEVERIANO, E. C.; MELLO, C. R. Dinâmica da resistência à penetração em Latossolo vermelho da microrregião de Goiânia, GO. Revista Brasileira de Engenharia Agrícola e Ambiental, Campina Grande, v. 11, n. 3, p.265-270, 2007.

ORTIGARA, C.; KOPPE, E.; LUZ, F. B. D.; BERTOLLO, A. M.; KAISER, D. R.; SILVA, V. R. D. Uso do solo e propriedades físico-mecânicas de Latossolo vermelho. Revista Brasileira de Ciência do Solo, Viçosa, v. 38, n. 2, p.619-626, 2014.

REISE, S. P.; WALLER, N. G.; COMREY, A. L. Factor analysis and scale revision. Psychological Assessment, Washington, v. 12, n. 3, p.287-297, 2000. 
SENE, M.; VEPRASKAS, M. J.; NADERMAN, G. C.; DENTON, H. P. Relationships of soil texture and structure to corn yield response to sub soiling. Soil Science Society of America Journal, Madison, v. 49, n. 2, p. 422-427, 1985.

SHARMA, S. Applied Multivariate Techniques. New York: John Wiley and Sons, 1996. $512 \mathrm{p}$.

SILVA, G. J.; MAIA, J. C. S.; BIANCHINI, A. Crescimento da parte aérea de plantas cultivadas em vaso, submetidas à irrigação subsuperficial e a diferentes graus de compactação de um Latossolo Vermelho-Escuro distrófico. Revista Brasileira de Ciência do Solo, Viçosa, v. 30, n. 1, p.31-40, 2006.

SILVA, G. J. Abordagens multivariadas na avaliação da resistência do solo à penetração em função da profundidade e manejos. 2012. 230 f. Tese (Doutorado em Agricultura Tropical) - Universidade Federal de Mato Grosso, Cuiabá, 2012.

SOUZA, Z. M; MARQUES JUNIOR, J.; PEREIRA G, T. Variabilidade espacial de atributos físicos do solo em diferentes formas do relevo sob cultivo de cana-de-açúcar. Revista Brasileira Ciência do Solo, Viçosa, v. 28, n. 6, p.937-944, 2004.

STELlUTI, M.; MAIORANA, M.; DE GIORGIO, D. Multivariate approach to evaluate the penetrometer resistance in different tillage systems. Soil Tillage Research, Amsterdam, v. 46, n. 3-4, p.145-151, 1998.

TARDIEU, F. Growth and functioning of roots and to root systems subjected to soil compaction towards a system with multiple signaling. Soil Tillage Research, Amsterdam, v. 30, n. 2-4, p.217-243, 1994.

TAYLOR, H. M.; ROBERSON, G. M.; PARKER JUNIOR, J. J. Soil strength-root penetration relations for medium to coarse-textured soil materials. Soil Science, New Brunswick, v. 102, n. 1, p.18-22, 1966.

TORMENA, C. A.; ROLOFF, G. Dinâmica da resistência à penetração de um solo sob plantio direto. Revista Brasileira de Ciência do Solo, Viçosa, v. 20, n. 3, p.333-339, 1996.

TORMENA, C. A.; ROLOFF, G.; SÁ, J. C. M. Propriedades físicas do solo sob plantio direto, influenciadas por calagem, preparo inicial e tráfego. Revista Brasileira Ciência do Solo, Viçosa, v. 22, n. 2, p.301-309, 1998.

TREIN, C. R.; COGO, N. P.; LEVIEN, R. Métodos de preparo do solo na cultura do milho e ressemeadura do trevo na rotação aveia+trevo/milho, após pastejo intensivo. Revista Brasileira de Ciência do Solo, Viçosa, v. 15, n. 1, p.105-111, 1991.

VALADÃO JÚNIOR, D. D.; BIANCHINI, A.; SILVA, G. J.; VALADÃO, F. C. A. Uso da técnica de componentes principais na física do solo. Cultura Agronômica, Ilha Solteira, v. 25, n. 2, p.117-130, 2016.

ZWICK, R.; VELICER, W. F. Comparison of five rules for determining the number of components to retain. Psychological Bulletin, Washington, v. 99, n. 3, p.432-442, 1986. 
WEIRICH NETO, P. H.; BORGHI, E.; SVERZUT, C. B.; MANTOVANI, E. C.; GOMIDE, R. L.; NEWES, W. L. C. Análise multivariada da resistência do solo à penetração sob plantio direto. Ciência Rural, Santa Maria, v. 36, n. 4. p.1186-1192, 2006. 